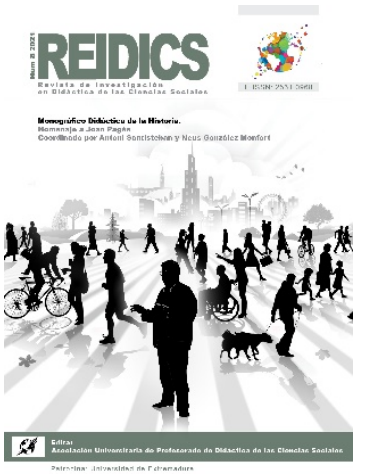

\title{
REIDICS
}

Revista de Investigación en

Didáctica de las Ciencias

Sociales
Núm. 8, 2021

Recibido 30 setiembre de 2020

Aceptado 25 de noviembre 2020

E-ISSN: 2531-0968

\section{El cuento en la enseñanza del patrimonio: análisis de propuestas didácticas en Educación Infantil}

The story in the teaching of heritage: Analysis of didactic proposals in Preschool Education

José María Cuenca López

Universidad de Huelva

Email: jcuenca@ddcc.uhu.es

ORCID: http://orcid.org/0000-0002-0190-5739

Marta Pérez González

Universidad de Huelva

Email: marta.pgonzalez@alu.uhu.es

ORCID: https://orcid.org/0000-0002-5892-3444

DOI: https://doi.org/10.17398/2531-0968.08.243

\section{Resumen}

La siguiente investigación consiste en el análisis de propuestas didácticas llevadas a cabo en el aula de infantil, las cuales utilizan el cuento como recurso educativo, con el objetivo de conocer el contenido patrimonial presente en las mismas. Una de sus finalidades es establecer unos parámetros para abordar una propuesta de educación patrimonial deseable y eficiente trabajando con cuentos en la etapa de Educación Infantil. Para ello, en primer lugar, se ha seleccionado una muestra de 10 propuestas didácticas para analizar, cuya información ha sido recabada mediante una rejilla de observación previamente elaborada. Posteriormente, esta información fue analizada con la ayuda de un sistema de categorías cualitativo. Por último esta información ha servido de referencia para establecer unos criterios adecuados para posibles propuestas de educación patrimonial.

Palabras clave: Educación Infantil; educación patrimonial; cuento infantil; propuestas didácticas y estrategias metodológicas.

\section{Abstract}

The following investigation consists of the analysis of didactic proposals carried out in the children's classroom, which require the story as an educational resource, with the aim of knowing the heritage content present in them. The main objective of the study is to establish 
parameters to address a desirable and efficient heritage education proposal working with stories in the Preschool Education. For this, in the first place, a sample of 10 didactic proposals for analysis has been selected, whose information has been collected through a previously prepared observation grid. Subsequently, this information was analyzed using a qualitative category system. Finally, this information has served as a reference to establish specific criteria for subsequent proposals for heritage education.

Keywords: Preschool Education; heritage education; children's story; didactic proposals and methodological strategies.

\section{Fundamentación teórica}

\subsection{La educación patrimonial en Educación Infantil}

¿Qué se entiende por patrimonio? Siguiendo a Cuenca (2016) no se puede dudar de la gran complejidad conceptual del término patrimonio y los muy diversos ámbitos que abarca.

Según Cuenca (2002), Fontal (2003) y Martín (2012), el concepto de patrimonio se ha vinculado con aspectos materiales. No obstante, cuando hacemos alusión al concepto de patrimonio, no solo se ha de relacionar con elementos materiales o con esos valores que las personas le atribuyen a los bienes, los cuales pueden ser emotivos, sociales, de uso, identitarios, etc., sino que también hacemos referencia a esas relaciones que se establecen entre los bienes y los seres humanos, como la identidad, la pertenencia y el sentimiento de propiedad.

En este sentido, Estepa, Domínguez y Cuenca (1998), afirman que los elementos patrimoniales potencian los referentes identitarios y simbólicos de la sociedad, al mismo tiempo que favorecen la tolerancia y el respeto hacia otras formas de vida pasadas o actuales, básicamente a través de la empatía cultural, valorando así la interculturalidad como condición imprescindible para un mundo mejor.

Según Arévalo (2004), el patrimonio se convierte en el vínculo entre generaciones, en lo que caracteriza e identifica la cultura de cada sociedad. Por tanto, no solo debe ser protegido por sus valores estéticos y de antigüedad, sino también por lo que significa y representa.

Así mismo, debido a su gran valor simbólico-identitario, no cabe duda lo imprescindible que es incluir la enseñanza del patrimonio en la escuela con el objetivo de formar ciudadanos activos y críticos, los cuales defiendan, protejan y valoren los elementos patrimoniales (costumbres, tradiciones, etc.) de su entorno.

Tras aclarar la necesidad de incluir contenidos patrimoniales en la escuela, trataremos de acercarnos al término "educación patrimonial", haciendo un recorrido por el currículo de la etapa educativa que nos concierne en esta investigación.

Teniendo presente a Fontal (2003), la "educación patrimonial" hace referencia a la acción educativa que pretende mantener y conservar los bienes patrimoniales, tanto heredados como adquiridos. Dichos bienes patrimoniales (monumentos, esculturas, obras literarias, danzas...) son concebidos como las huellas de las sociedades, las cuales han estado presente durante el paso de la historia, por lo que la convivencia con estos bienes ha sido constante, aunque no siempre fácil. 
Siguiendo a Cuenca y Martín (2014) la enseñanza del patrimonio ha de estar presente en el proceso educativo como una meta de la educación, concretamente, en el ámbito de la formación ciudadana. Por consiguiente, el patrimonio debe ser trabajado como contenido conceptual, procedimental, y actitudinal, no solo ser considerado como un recurso u objeto de trabajo.

Se presenta como imprescindible la inclusión de contenidos patrimoniales en los centros educativos debido al desconocimiento y desinterés que las personas presentan frente al patrimonio, lo cual conlleva a la paulatina destrucción y desaparición del patrimonio (Guillén y Hernández, 2018). Se ha de lograr la concienciación patrimonial, implicando a la sociedad en el conocimiento y valoración del patrimonio, puesto que la conservación del mismo, depende mayoritariamente de la valoración que la sociedad haga del mismo (Casanova, Arias y Egea, 2018).

Gracias a la enseñanza-aprendizaje de elementos patrimoniales, el alumnado logra el conocimiento del pasado, la comprensión del presente, y el origen del futuro próximo; y también adquiere nociones espaciales y temporales, mejora su pensamiento crítico, la empatía, la solidaridad, la cooperación y el respeto hacia otras culturas. Estos son algunos de los motivos por los que se considera de vital importancia la educación patrimonial en la escuela (Estepa, Domínguez y Cuenca, 1998).

Tal y como se ha comentado, el patrimonio se concibe como un símbolo identitario, cuya integración en el currículo puede hacer progresar la educación de los alumnos y alumnas en la escuela, fomentado el desarrollo de un pensamiento más completo y un conocimiento patrimonial deseable (Ávila, 2005).

Centrándonos en el currículo de Educación Infantil, el concepto de "educación patrimonial" no aparece de forma explícita en éste. Según Miralles y Rivero (2012), solo aparece de manera transversal en diversos bloques de contenidos. No obstante, sí acoge la cultura andaluza (la cual forma parte del patrimonio) como un contenido que se ha de enseñar en las escuelas. Concretamente, se hace referencia a este tipo de patrimonio en uno de los objetivos generales de la etapa: “Conocer y participar en manifestaciones culturales y artísticas de su entorno, teniendo en cuenta su diversidad y desarrollando actitudes de interés, aprecio y respeto hacia la cultura andaluza y la pluralidad cultural" (Orden del 5 de agosto de 2008) ${ }^{1}$.

Por otro lado, haciendo referencia a una de las áreas del currículo "Conocimiento del Entorno", en esta también se hace alusión a contenidos patrimoniales: "Progresivamente se han de ir acercando al conocimiento de algunos rasgos culturales propios. La diversidad cultural aconseja aproximar a niños y niñas a los usos y costumbres sociales de una perspectiva abierta e integradora que les permita conocer diversos modos y manifestaciones culturales presente en la sociedad, y generar así actitudes de respeto y aprecio hacia ellas" (Real Decreto 1630/2006, de 29 de diciembre) $)^{2}$.

No obstante, al no estar explícitamente incorporado el término de patrimonio en el currículo educativo, la inclusión de este tipo de contenidos queda a merced del docente (Casanova, Arias y Egea 2018), los cuales tienen muy arraigada la enseñanza tradicional basada en la

1 Orden del 5 de agosto de 2008, por la que se desarrolla el Currículo correspondiente a la Educación Infantil en Andalucía.

2 Real Decreto 1630/2006, de 29 de diciembre por el que se establece las enseñanzas mínimas del segundo ciclo de Educación Infantil. 
fragmentación de contenidos para su enseñanza (Ávila, 2005). Es decir, dichas legislaciones no especifican la metodología adecuada para trabajar este contenido en los centros escolares.

En este sentido, siguiendo a Casanova, Arias y Egea (2018), el principal problema que existe en Educación Infantil es el cómo trabajar este tipo de contenidos en el aula. De este modo, dichos autores afirman que los obstáculos presentes a la hora de trabajar contenidos patrimoniales en infantil se deben más a la metodología a emplear (estrategias, recursos, metodología) que a las propias capacidades cognitivas del alumnado.

\subsection{El cuento infantil en el proceso de enseñanza-aprendizaje}

Debido a la problemática comentada, en este apartado nos vamos a centrar en la explicación de los cuentos infantiles, como recursos motivadores y competentes para la enseñanza de contenidos patrimoniales en edades tempranas.

Como antecedente, y considerando el patrimonio como un contenido transversal, González (2006) en su investigación se hace la siguiente pregunta: ¿Qué estrategias podemos emplear para trabajar contenidos transversales?, obteniendo como resultado lo siguiente: "una de las principales respuestas que aportan los profesionales de la educación es el cuento, dada su importancia demostrada en el desarrollo de hábitos y destrezas, así como su contribución al estímulo de las competencias de la infancia y la adolescencia" (González, 2006, p.12). Por otro lado, varios autores hablan de la gran relevancia que tiene el cuento en el desarrollo psicológico infantil, así como en la transmisión de la herencia cultural (León, 2009).

Como afirma Ramón (2004, p. 149), “el cuento es aparentemente el más antiguo de los géneros, puesto que se cultiva antes de que se tuviera conciencia de él como género literario". Por consiguiente, el cuento es probablemente el género literario que antes se inventó, aunque simplemente fuese transmitido de manera oral. Pero, además, si hablamos de las narrativas dirigidas al público infantil, el cuento se presenta como el género más predominante.

Tal y como recoge Salmerón (2004) en sus Tesis Doctoral, el cuento clásico es considerado como una de las principales herramientas culturales que ayudan a los más pequeños a conocer el mundo en sus primeros años de vida. Los niños y las niñas están en contacto con libros infantiles desde su nacimiento, puesto que forman parte del repertorio cultural que las generaciones adultas han transmitido oralmente y siguen transmitiendo a los más jóvenes (Morón, 2010).

Según Jiménez y Gordo (2014) y Morón (2010), la recepción de literatura desde la primera infancia es la actividad más beneficiosa para conseguir el éxito de los niños y niñas en su acceso a la comunicación escrita. Además, favorece la construcción de su identidad y la del entorno que le rodea, y fomenta el desarrollo afectivo, emocional y social de los más pequeños, al influir tanto en el enfoque afectivo como en el cognitivo, favoreciendo la imaginación y permitiendo una total divergencia del pensamiento (Franco y Justo (2009).

Siguiendo en esta misma línea, Ros (2013) aporta que el cuento muestra imágenes simbólicas con la finalidad de explicar el mundo social, siendo un instrumento socializador transmisor de los valores y los usos de una comunidad, siendo su función tanto educativa como de ocio. 
Por tanto, los cuentos no solo divierten y entretienen, también transmiten costumbres, tradiciones, creencias y valores, que ayudan a la integración del niño y la niña en la sociedad, ambiente y momento histórico en el que se encuadran. Además, fomentan la imaginación de estos, la cual funciona como base del pensamiento y del lenguaje, dando lugar a la posibilidad de reavivar y evocar acontecimientos del pasado (Morón, 2010 y Fernández, 2010).

Por otro lado, siguiendo a Amar (2018), el cuento se presenta como un referente esperado por los niños y las niñas, convirtiéndose en un recurso primordial a ser usado en el aula por los docentes. Es decir, "el cuento es un momento álgido y mágico donde el silencio se apodera de la acción del aula" (p. 391), da igual en que momento del día, el silencio y la participación se apodera de la clase.

En cuanto a los tipos de cuentos (Tabla 1), debido a su gran variedad, se ha seleccionado la clasificación de Pelegrín (1982) y Rodríguez (1989), citados en Salmerón (2004). Cabe destacar que esta clasificación se ha adoptado como referencia para el análisis de la potencialidad didáctica de los cuentos infantiles.

Tabla 1

Tipos de cuentos.

Cuentos rimados y de fórmula: Cuentos mínimos y breves, los de nunca acabar y acumulativos.

Cuentos de animales: Los protagonistas son animales y están personificados.

Cuentos maravillosos o de hadas: Intervienen aspectos mágicos o sobrenaturales y los personajes tienen características irreales.

Fábulas: Los personajes son animales que actúan como personas. Se diferencian de los cuentos de animales porque terminan con una moraleja.

Leyendas: Tratamiento fantástico y mágico de hechos relativamente reales.

Relatos de historia natural: Relata hechos científicos naturales basados en las plantas, animales, fenómenos atmosféricos,...

Cuentos de costumbres: Reflejan modos de vida en un determinado lugar y momento, contado la mayoría de las veces en tono irónico y burlón.

Fuente: Elaboración propia basada en Pelegrín (1982) y Rodríguez (1989).

Por todo lo comentado, no podemos dudar de su valor educativo ya que, confirmando las palabras de Quintero (2005), el cuento infantil:

- Sirve para divertir y entretener a la vez que transmiten conocimientos ricos y complejos.

- Satisface las ganas de acción del alumnado puesto que en su imaginación proyectan lo que les gustaría hacer.

- Conecta con las características cognitivo- afectivas de niños y niñas. La narración del cuento enlazará rápidamente con el mundo interno del niño/a, contribuyendo al desarrollo de su capacidad simbólica.

- Es un elemento socializador que favorece las relaciones, empezando por el simple hecho de que son los propios personajes los que interactúan socialmente.

- Favorece el desarrollo de la empatía y la superación del egocentrismo al ponerse en el lugar de los diferentes protagonistas, lo cual extrapola a su vida cotidiana.

- Prepara para la vida, ofreciendo modelos de comportamiento, sentimientos y valores. 
En esta misma línea, es decir, cómo otro recurso tradicional para el fomento del patrimonio tanto material como inmaterial, se destaca las canciones tradicionales. Según Díaz y Arriaga (2013) llevar al aula las canciones tradicionales infantiles supone mantener vivo el patrimonio artístico y cultural de nuestras sociedades, pues se presentan como un elemento primordial en la educación, las cuales proporcionan placeres en los diversos momentos de aprendizaje e interpretación. Es decir, los niños y las niñas aprenden educación musical y patrimonial, a la vez que se divierten con las canciones.

Según esta investigación, la mayoría de los docentes encuestados consideran las canciones tradicionales como una forma adecuada de establecer contacto con nuestras costumbres y antepasados. Es decir, las canciones tradicionales se contemplan como un recurso didáctico adecuado para la enseñanza de contenidos patrimoniales. Además, es un recurso significativo y motivador para los niños y las niñas, gracias al cual se trabajan diversos contenidos como la educación patrimonial, la educación musical, o la lecto-escritura, así como otros aspectos de carácter transversal (la atención, la memoria, la imaginación, la creatividad, etc.), los cuales emanan de la utilización de este recurso. Así mismo, se contemplan a los cuentos infantiles, como un recurso motivador y cercano al alumnado, el cual permite la enseñanza globalizada de muchos contenidos.

La utilización de los cuentos infantiles en el aula debe de ir dentro de una propuesta didáctica adecuada y adaptada a las características de la etapa educativa pertinente, con el objetivo de obtener en el alumnado el mayor rendimiento académico posible. Siguiendo el currículo de Educación Infantil, una práctica educativa tiene que perseguir el objetivo de contribuir al desarrollo físico, afectivo, social e intelectual de los niños/as, respetando los derechos de la infancia y atendiendo a su bienestar.

En este sentido, los contenidos a trabajar en estas propuestas se orientan hacia la consecución del desarrollo de los distintos planos que integran la personalidad infantil: físico y motórico, afectivo, lingüístico, social y cognitivo. Dichos aspectos se han de integrar de manera globalizadora, por lo que será necesario incluir contenidos patrimoniales de forma interdisciplinar y holística, empleando unidades didácticas donde la idea de patrimonio se perciba de manera integrada (Ávila, 2005).

Además, Miralles y Rivero (2012) lanzan una serie de propuestas metodológicas a tener en cuenta en la planificación de la enseñanza y aprendizaje de contenidos sociales en Educación Infantil, las cuales vamos a unificar en Tabla 2.

Por último, con respecto a la evaluación, esta debe tener un carácter global, procesual y continuo, ya que ha de estar presente en el desarrollo de todo tipo de actividades y no solo en momentos puntuales y aislados. Además, se evaluará tanto el proceso de enseñanza como el de aprendizaje, es decir, no solo la evolución de nuestros alumnos/as, sino también nuestra practica educativa. Como instrumentos de evaluación, el currículo destaca la observación y los diarios de clase, con el propósito de elaborar documentación narrativa sobre la práctica docente y las experiencias de aula. Los criterios a evaluar deben ser establecidos por cada tutor/a, teniendo en cuenta los objetivos a conseguir en la práctica educativa. 
Tabla 2

Propuestas metodológicas.

\begin{tabular}{ll}
\hline & -Aprendizaje significativo. \\
Estrategias metodológicas & -Metodología intuitiva. \\
& -Enfoque globalizador. \\
& -Aprendizaje por descubrimiento. \\
& -Participación familiar. \\
& -Importancia de la narración. \\
& -Programación de salidas del aula. \\
\hline \multirow{2}{*}{ Papel del docente } & -No ha de ser el transmisor de los contenidos. \\
& -Facilitador del aprendizaje. \\
& -Guía y modelo. \\
& -Fomentar un clima afectivo en el aula. \\
\hline & -Papel principal en el aula. \\
Papel del alumnado & -Protagonista de su propio aprendizaje. \\
\hline
\end{tabular}

Fuente: Elaboración propia, basada en Miralles y Rivero (2012).

\section{Marco metodológico}

La presente investigación se ha basado en el análisis de propuestas didácticas llevadas a cabo en el aula de infantil, las cuales utilizan el cuento como recurso educativo, con el objetivo de conocer el contenido patrimonial presente en las mismas. Para ello se ha seleccionado un método de investigación cualitativo, concretamente el "estudio documental". Siguiendo a Bisquerra (2014), el análisis documental es una actividad sistemática y planificada que consiste en examinar documentos ya escritos que abarcan una amplia gama de modalidades. A través de ellos es posible captar información valiosa.

\subsection{Pregunta de investigación y objetivos}

Tal y como se ha comentado, Casanova, Arias y Egea (2018), perciben que el principal problema que existe en Educación Infantil es el cómo trabajar contenidos transversales en el aula, como en nuestro caso, el patrimonio.

De esta problemática, surge la siguiente pregunta, a la que damos respuesta con el diseño metodológico del estudio: ¿Qué criterios se deben de tener en cuenta para desarrollar propuestas de educación patrimonial deseables utilizando los cuentos como recurso didáctico en el aula de 3 a 5 años? Esta sería la pregunta de investigación a la que se pretende dar respuesta en esta investigación. Por consiguiente, los objetivos que se plantean son los siguientes:

Objetivo general:

- Examinar diferentes propuestas didácticas, correspondientes a la etapa de Educación Infantil, las cuales usan el cuento como instrumento de enseñanza.

Objetivos específicos:

- Destacar la finalidad didáctica más frecuente en las propuestas didácticas.

- Detectar los métodos y estrategias utilizadas en las propuestas didácticas.

- Conocer qué tipos de actividades son las más habituales en las propuestas didácticas. 
- Indagar sobre el papel del docente y del alumnado en el trascurso de las propuestas didácticas.

- Determinar el proceso evaluativo llevado a cabo en las propuestas didácticas.

- Profundizar sobre el papel del patrimonio presente en las propuestas didácticas.

- Averiguar qué tipo de patrimonio se trabaja en las propuestas didácticas.

- Conocer los tipos de cuentos más usados en las propuestas didácticas.

- Establecer unos parámetros para abordar una propuesta de educación patrimonial deseable y eficiente trabajando con cuentos en la etapa de Educación Infantil.

\subsection{Materiales}

La muestra investigada ha sido 10 propuestas didácticas (Tabla 3), las cuales se han seleccionado de diferentes bases de datos, siguiendo dos criterios: por un lado, propuestas didácticas en las que se aborde algún contenido patrimonial, y por otro lado, propuestas didácticas que utilicen algún tipo de cuento infantil. Estas propuestas didácticas trabajan diversos contenidos curriculares de manera globalizadora, aunque cada una de ellas se centra en un objetivo específico (lecto-escritura, matemáticas, interculturalidad, historia, obras artísticas, entre otros).

Tabla 3

Propuestas didácticas analizadas.

\begin{tabular}{ll}
\hline PROPUESTAS DIDÁCTICAS & CUENTOS \\
1. Parque de la Alquería del Pilar & Cuento sobre el parque (elaboración docente) \\
2. Príncipes y princesas & El príncipe Encantado \\
3. Aprendizaje lógico-matemático & El lobo y los siete cabritillos \\
4. Educación intercultural & Bruno (elaboración docente) \\
5. Aprendemos sobre Paul klee & Elmer \\
6. Iniciación a la lecto-escritura & Cuentos clásicos \\
7. Pintamos nuestro patrimonio & La Giralda \\
8. ¿Iguales o diferentes? & Cirilo, el cocodrilo \\
9. Aprendemos matemáticas & Los siete cabritillos \\
10. iGarras está en peligro! & Aurora, una pequeña lince en Doñana \\
\hline
\end{tabular}

Fuente: Elaboración propia.

\subsection{Instrumentos}

Para recoger la información que dará respuesta a la pregunta de investigación y permitirá alcanzar los objetivos propuestos, se ha elaborado una rejilla de observación y un sistema de categorías.

Ambos instrumentos cuentan con una serie de ítems a analizar clasificados en categorías y subcategorías. Estos ítems se corresponden con las diferentes partes que suele tener una propuesta didáctica (objetivos, competencias, contenidos, metodología, evaluación y cuentos 
utilizados), así como con aspectos relacionados con la educación patrimonial (tipos de patrimonio y su papel). De este modo, nos permite indagar sobre las características curriculares y didácticas que poseen las propuestas didácticas, la presencia de contenido patrimonial en la misma, y los tipos de cuentos más utilizados.

Además de estos ítems, la Tabla 5 de categorías contiene unos indicadores para facilitar el análisis de las propuestas. Con respecto a la validación de ambos instrumentos, se ha realizado una prueba piloto con una de las propuestas didácticas seleccionadas, concretamente, la propuesta número 9: "Aprendemos matemáticas", la cual utiliza el cuento clásico "Los siete cabritillos".

Tabla 4

Rejilla de observación

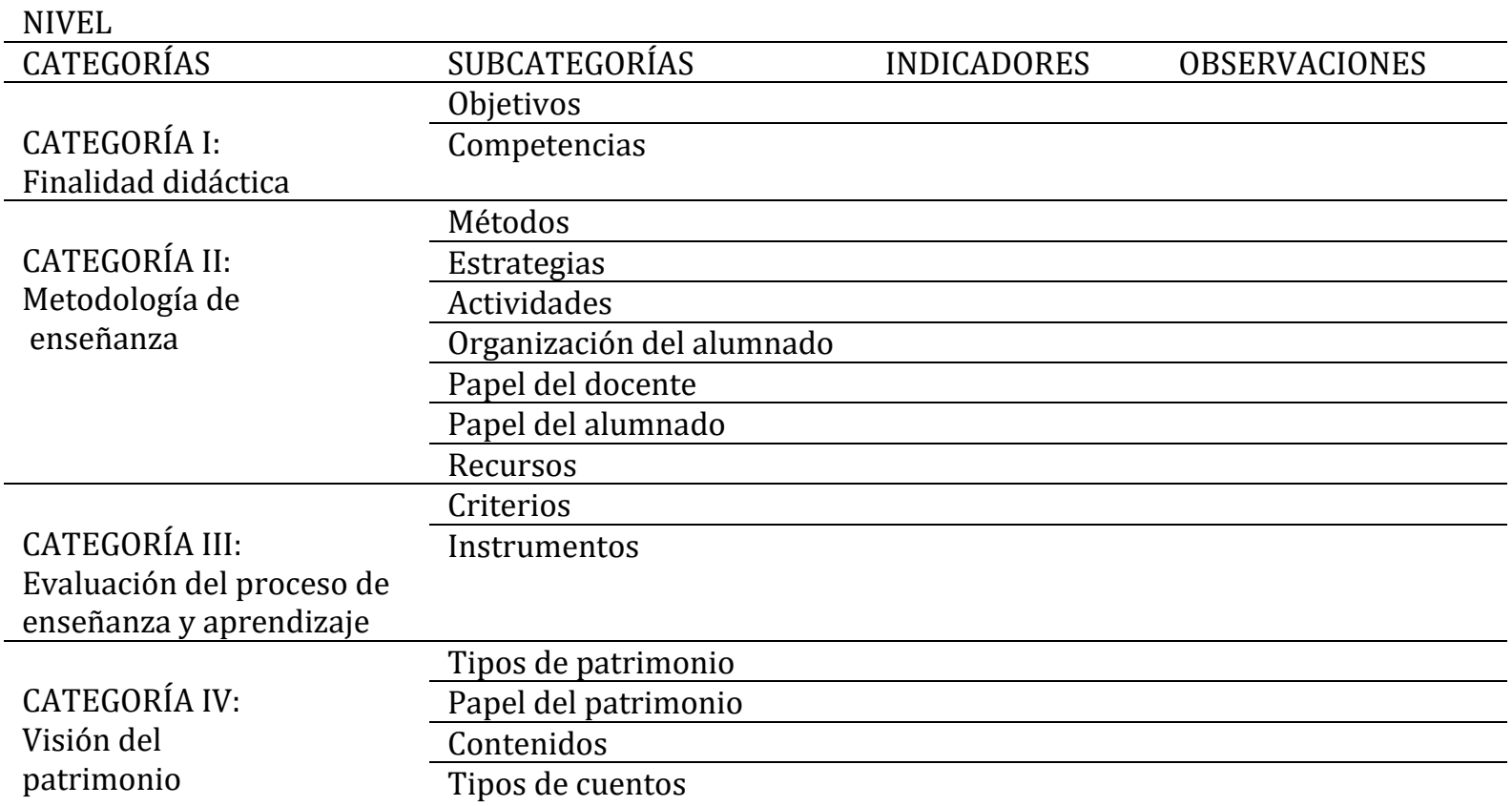

Fuente: elaboración propia.

Tabla 5

Sistema de categorías.

CATEGORÍAS

CATEGORÍA I:

Finalidad didáctica
SUBCATEGORÍAS INDICADORES

Construir su propia identidad e ir formándose una imagen positiva de sí mismo

Adquirir autonomía en la realización de

Objetivos actividades

Establecer relaciones sociales satisfactorias Observar y explorar su entorno físico, natural, social y cultural

Comprender y representar nociones y relaciones lógicas y matemáticas

Representar aspectos de la realidad vivida e imaginada

Utilizar el lenguaje oral para comprender y ser comprendido por los demás

Aproximarse a la lectura y escritura en situaciones de la vida cotidiana

Conocer y participar en manifestaciones 
CATEGORÍA II:

Metodología de enseñanza culturales y artísticas de su entorno

\begin{tabular}{|c|c|}
\hline \multirow{7}{*}{$\begin{array}{l}\text { Competencias } \\
\text { claves }\end{array}$} & Lingüística \\
\hline & Matemática \\
\hline & Digital \\
\hline & Aprender a aprender \\
\hline & Sociales y cívicas \\
\hline & Sentido de la iniciativa y espíritu emprendedor \\
\hline & Conciencia y expresiones culturales \\
\hline \multirow{4}{*}{ Métodos } & Tradicional \\
\hline & Aprendizaje basado en proyectos \\
\hline & Aprendizaje cooperativo \\
\hline & Trabajo por rincones \\
\hline \multirow{4}{*}{ Estrategias } & Aprendizaje significativo \\
\hline & Enfoque globalizador \\
\hline & Aprendizaje por descubrimiento \\
\hline & Participación familiar \\
\hline \multirow{5}{*}{ Actividades } & Previas y de motivación \\
\hline & De desarrollo \\
\hline & Finales \\
\hline & De refuerzo y ampliación \\
\hline & De evaluación \\
\hline \multirow{4}{*}{$\begin{array}{l}\text { Organización del } \\
\text { alumnado }\end{array}$} & Individual \\
\hline & Parejas \\
\hline & Pequeños grupos \\
\hline & Gran grupo \\
\hline \multirow[b]{2}{*}{ Papel docente } & Activo \\
\hline & Pasivo \\
\hline \multirow{2}{*}{ Papel alumnado } & Activo \\
\hline & Pasivo \\
\hline \multirow{4}{*}{ Recursos } & Materiales \\
\hline & Informáticos \\
\hline & Humanos \\
\hline & Espaciales \\
\hline \multirow{12}{*}{ Criterios } & Control corporal y destrezas motoras \\
\hline & Autonomía personal \\
\hline & Hábitos de higiene personal \\
\hline & Socialización con los iguales \\
\hline & Reconocimiento y control de las emociones \\
\hline & $\begin{array}{l}\text { Conocimiento de objetos y elementos del } \\
\text { entorno inmediato }\end{array}$ \\
\hline & $\begin{array}{l}\text { Actitudes de cuidado y respeto hacia la } \\
\text { naturaleza }\end{array}$ \\
\hline & Comprensión de manifestaciones culturales \\
\hline & Iniciación en la lecto-escritura \\
\hline & Habilidades matemáticas \\
\hline & Capacidad de comprensión a los demás \\
\hline & Utilización del lenguaje oral \\
\hline \multirow{5}{*}{ Instrumentos } & Observación/ficha de observación \\
\hline & Diario del profesor \\
\hline & Producciones del alumnado \\
\hline & Fichas de autoevaluación \\
\hline & Rubrica \\
\hline \multirow{4}{*}{ Tipos patrimonio } & Natural \\
\hline & Histórico \\
\hline & Científico-tecnológico \\
\hline & Etnológico \\
\hline
\end{tabular}

CATEGORÍA III:

Evaluación del proceso de enseñanza y aprendizaje 
Visión del patrimonio

\begin{tabular}{|c|c|}
\hline & Holístico \\
\hline \multirow{3}{*}{ Papel patrimonio } & Recurso didáctico \\
\hline & Transversal \\
\hline & Integración plena \\
\hline \multirow{9}{*}{ Contenidos } & La identidad personal, el cuerpo y los demás \\
\hline & Vida cotidiana, autonomía y juego \\
\hline & Medio físico: elementos, relaciones y medidas \\
\hline & Acercamiento a la naturaleza \\
\hline & Vida en sociedad y cultura \\
\hline & Lenguaje corporal \\
\hline & Lenguaje verbal \\
\hline & Lenguaje artístico: musical y plástico \\
\hline & Lenguaje audiovisual y las TIC \\
\hline \multirow{7}{*}{ Tipos de cuentos } & Cuentos rimados y de formula \\
\hline & Cuentos de animales \\
\hline & Cuentos maravillosos o de hadas \\
\hline & Fábulas \\
\hline & Leyendas \\
\hline & Relatos de historia natural \\
\hline & Cuentos d \\
\hline
\end{tabular}

Fuente: elaboración propia basada en Ferreras (2011).

\section{Resultados del estudio}

Para extraer resultados de la información recabada mediante las rejillas de observación (Tabla 4), nos basamos en el sistema de categorías planteado (Tabla 5).

\subsection{Categoría I: Finalidad didáctica}

En cuanto a la finalidad didáctica de las propuestas analizadas, nos centramos en sus objetivos y competencias claves. Con respecto al objetivo principal de cada propuesta didáctica, cabe destacar lo siguiente: dos propuestas contemplan como finalidad las matemáticas; otras dos propuestas, la educación intercultural; y otras dos, el patrimonio cercano; y las cinco propuestas restantes, cada una de ellas, se centran en un objetivo: lecto-escritura, príncipes y princesas, medioambiente y manifestaciones artísticas (Figura 1).

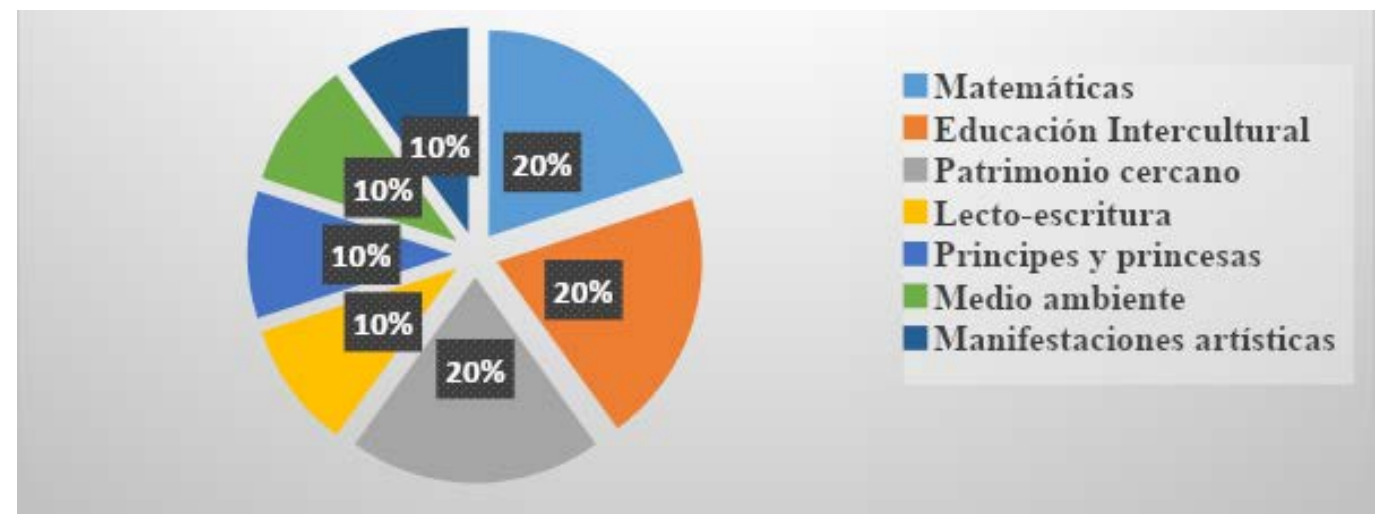

Figura 1. Objetivos principfales de las propuestas didácticas.

Fuente: elaboración propia. 
Además de tener un objetivo principal, las propuestas didácticas también contemplan otros objetivos curriculares. En este sentido, la mayoría de estas comparten los siguientes: Adquirir autonomía en la realización de actividades, establecer relaciones sociales satisfactorias, observar y explorar su entorno físico, natural, social y cultural, y aproximarse a la lectura y escritura en situaciones de la vida cotidiana. Así mismo, seis de las propuestas abarcan el objetivo curricular relacionado con el patrimonio: Conocer y participar en manifestaciones culturales y artísticas de su entorno.

Haciendo referencia a las competencias claves, todas las propuestas recogen la competencia lingüística, y social y cívica como parte de su finalidad didáctica; y solo tres propuestas contemplan la competencia matemática, digital y aprender a aprender. Cabe destacar que seis de las diez propuestas abarcan la competencia de conciencia y expresiones culturales, competencia relacionada con el tema en cuestión.

\subsection{Categoría II: Metodología de enseñanza}

Esta categoría hace alusión a la metodología de enseñanza desempeñada en cada una de las propuestas didácticas. Para ello, se ha tenido en cuenta los métodos, las estrategias, las actividades, la organización del alumnado, los recursos empleados en las mismas, así como el papel que se le otorga tanto al docente como al discente.

En primer lugar, en cuanto a los métodos empleados (Figura 2), el tradicional se presenta en cuatro propuestas, el Aprendizaje Basado en Proyectos (ABP) en ocho propuestas, el aprendizaje cooperativo en dos, y el trabajo por rincones solo en una de las propuestas didácticas. En este sentido, cabe destacar la presencia de tres propuestas, las cuales combinan aspectos característicos del método tradicional y del ABP, pues aúnan la investigación del alumnado con la realización de fichas.

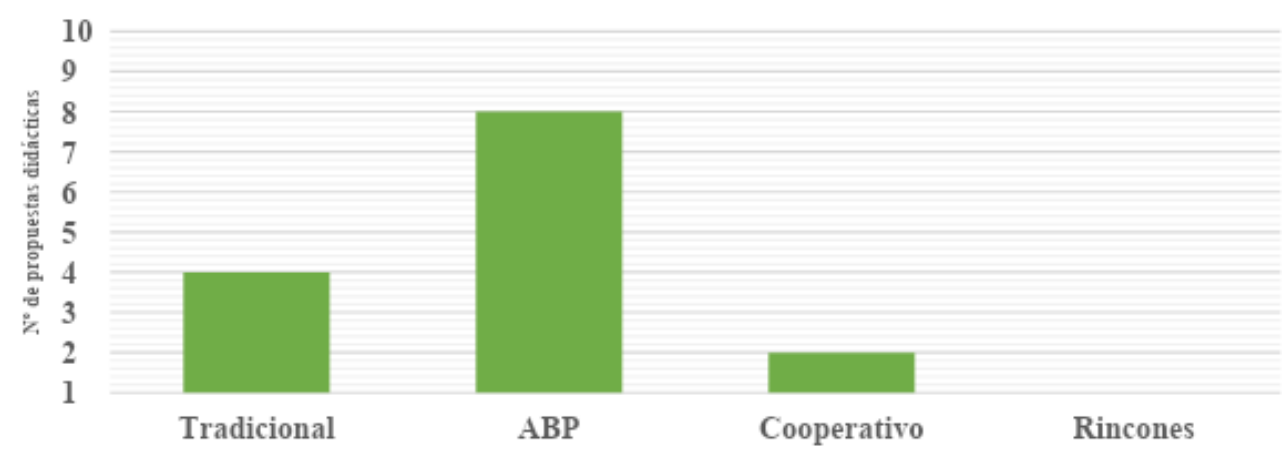

Figura 2: Métodos utilizados en las propuestas didácticas.

Fuente: elaboración propia.

En segundo lugar, con respecto a las estrategias metodológicas (Figura 3), el aprendizaje significativo y el enfoque globalizador se contemplan en todas las propuestas. Por otro lado, la participación familiar y el aprendizaje por descubrimiento solo se observan en cuatro propuestas. 


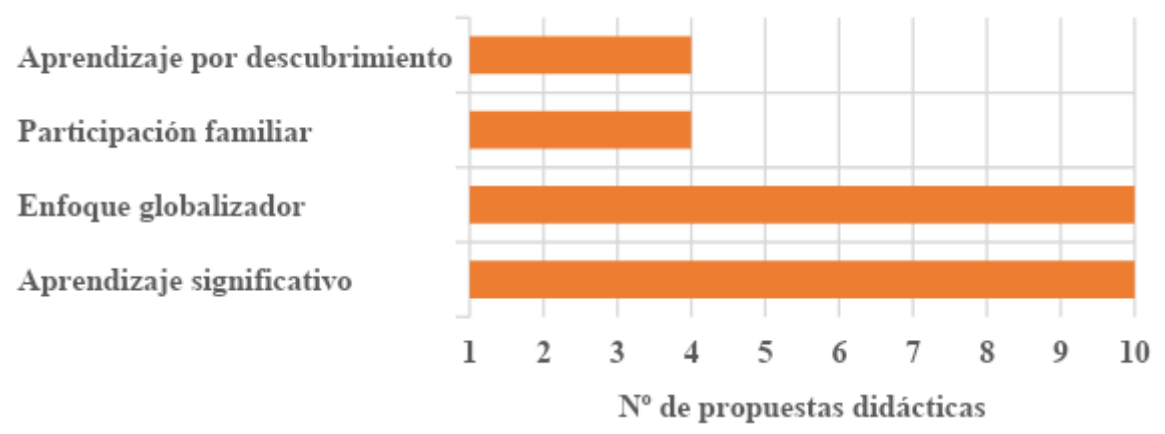

Figura 3: Estrategias metodológicas.

Fuente: elaboración propia.

En tercer lugar, en el caso de las actividades planificadas, en todas las propuestas nos encontramos con actividades previas y de motivación, y de desarrollo. Las actividades finales están presentes en siete propuestas didácticas, mientras que las actividades de evaluación solo se observan en cuatro propuestas. No obstante, no en todas las propuestas analizadas, se les designa a las actividades de esta manera. Por otro lado, cabe destacar la no presencia de actividades de refuerzo y ampliación en ninguna de las propuestas analizadas.

Centrándonos en las actividades destinadas para la enseñanza de elementos patrimoniales, nos encontramos con diversos tipos. Por un lado, la asamblea se presenta como la actividad más utilizada en las propuestas didácticas. Y por otro lado, la búsqueda de información, por parte del alumnado, sobre elementos patrimoniales se contempla en cuatro propuestas, la visita o excursión a elementos patrimoniales en tres propuestas, y la práctica de juegos populares o fiestas culturales, así como la creación de obras artísticas en otras cuatros propuestas.

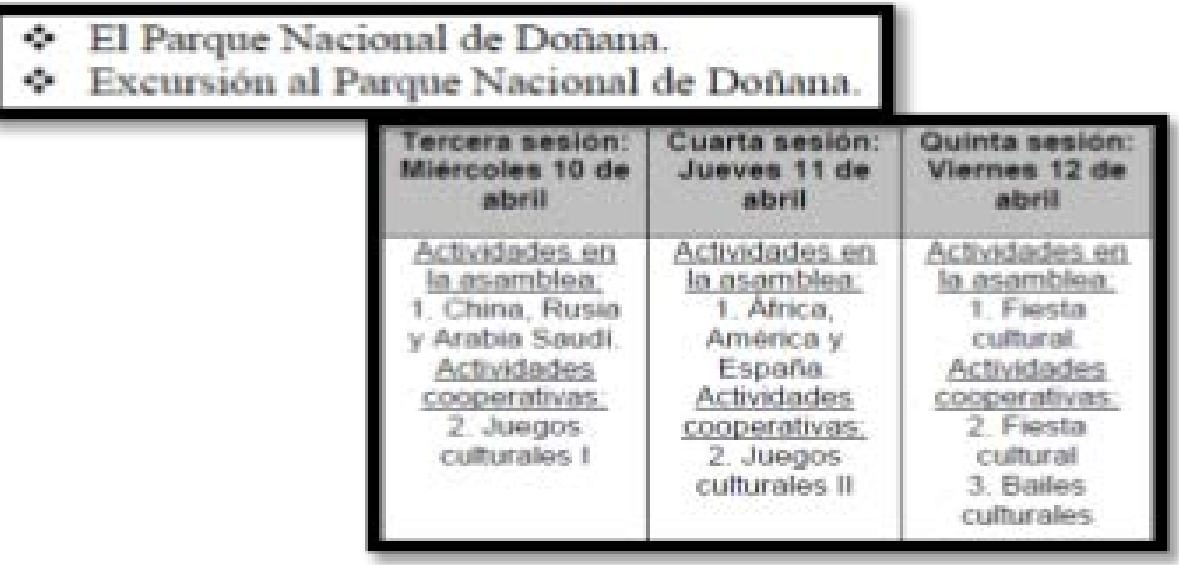

Figura 4. Ejemplos de actividades planificadas.

Fuente: Propuesta didáctica 9, página 2, y propuesta 4, página 194.

En cuarto lugar, haciendo referencia a la organización del alumnado, en todas las propuestas didácticas se observa la organización en gran grupo y en pequeños grupos durante la puesta en práctica de las actividades. No obstante, en la mayoría de ellas, la realización de la tarea se lleva a cabo de manera individual, menos en tres propuestas que se trabaja mediante técnicas de 
cooperativo. En esta subcategoría, cabe destacar la organización del alumnado durante las actividades relacionadas con contenidos patrimoniales, siendo la disposición en gran grupo la más utilizada en estas actividades.

En quinto lugar, con respecto al papel desempeñado por los docentes y por los discentes (Tabla 6), los resultados han sido variados y muy difíciles de identificar, pues hay una gran brecha entre la teoría y la práctica.

Por consiguiente, cabe destacar la falta de información en dos propuestas didácticas, por lo que, en esta subcategoría solo se han obtenido datos de ocho propuestas. Dicho esto, en la mayoría de ellas, el papel que se observa es pasivo, por parte del docente, y un papel activo, correspondiente a los discentes.

Tabla 6

Papel de los docentes y discentes.

\begin{tabular}{cccc}
\hline & ACTIVO & PASIVO & activo/pasivo \\
\cline { 2 - 4 } & & $N^{o}$ de propuestas & \\
\hline Docentes & 1 & 5 & 2 \\
Discentes & 6 & 2 & 3 \\
\hline
\end{tabular}

Fuente: elaboración propia.

Y, por último, en cuanto a los recursos empleados (Figura 5) durante las actividades, los más utilizados son los recursos materiales, los humanos y los espaciales. Los recursos materiales y espaciales se utilizan en todas las propuestas, los recursos humanos están presentes en ocho propuestas, y los recursos informáticos solo se utilizan en tres propuestas. A continuación, se muestra algunos de los recursos utilizados en las propuestas didácticas analizadas:

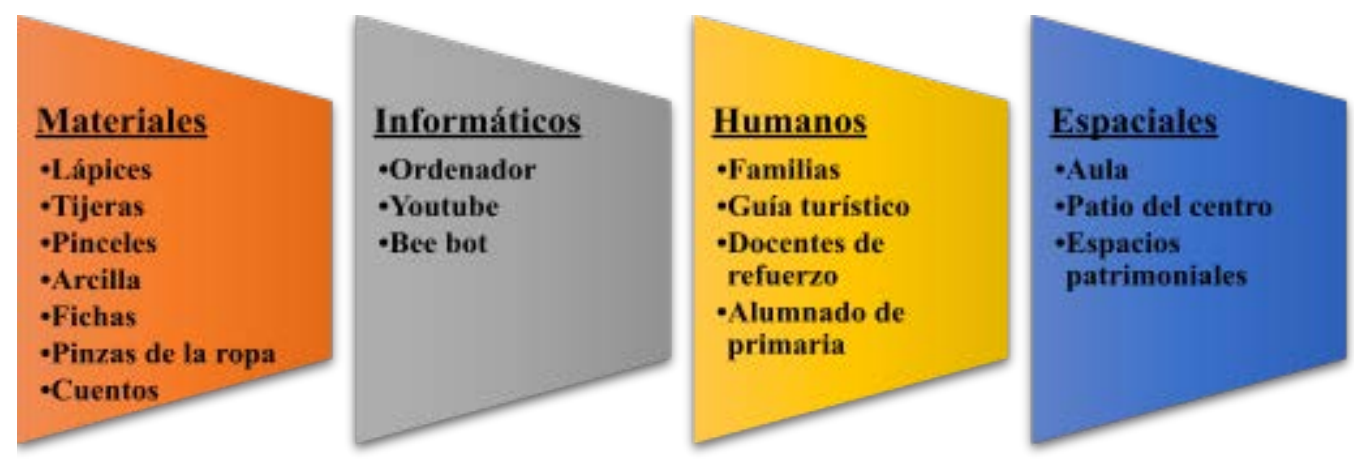

Figura 5. Tipos de materiales utilizados.

Fuente: elaboración propia.

Haciendo referencia a los recursos empleados para el tratamiento de contenidos patrimoniales, cabe destacar la utilización del ordenador e internet para buscar información de los mismos, el uso de espacios patrimoniales, y la ayuda de los guías turísticos para la explicación de estos espacios. 


\subsection{Categoría III: Evaluación del proceso de enseñanza-aprendizaje}

Todas las propuestas seleccionan sus criterios de evaluación en función a los objetivos planteados, siendo los más destacables: Autonomía personal, socialización con los iguales, conocimiento de objetos y elementos del entorno inmediato e iniciación a la lecto-escritura. Además, seis de las diez propuestas contemplan el criterio de evaluación sobre la comprensión de manifestaciones culturales.

En cuanto a los instrumentos de evaluación (Figura 6), todas las propuestas didácticas optan por la observación para evaluar el proceso de enseñanza-aprendizaje; ocho de estas diez propuestas, añaden a la observación, las producciones del alumnado; solo una propuesta recoge la rúbrica y el diario del profesor/a; y ninguna de las propuestas contemplan la autoevaluación.

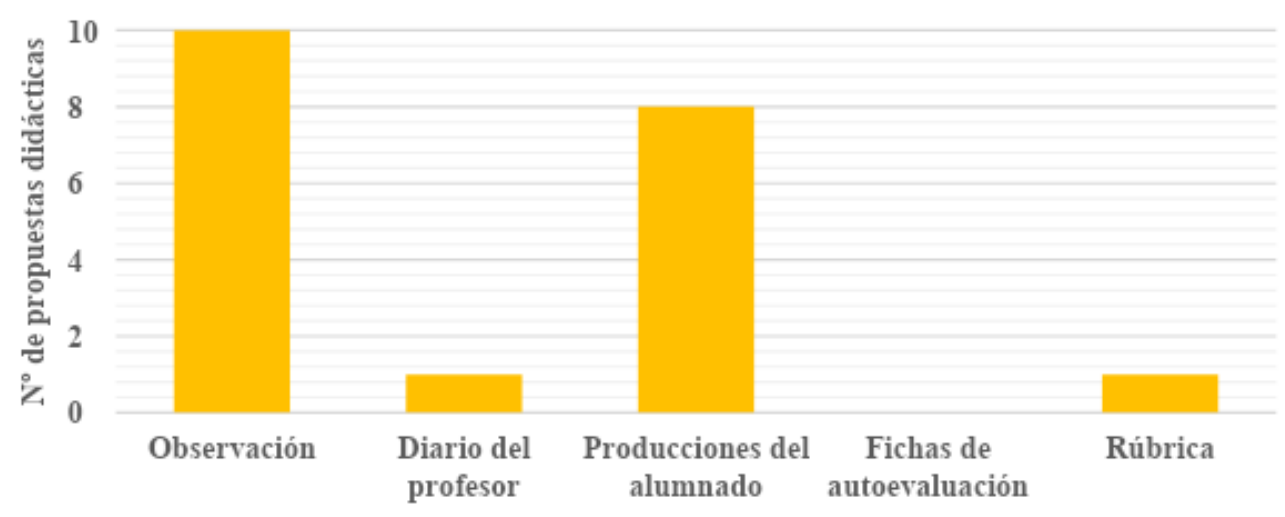

Figura 6. Instrumentos de evaluación.

Fuente: elaboración propia.

\subsection{Categoría IV: Visión del patrimonio}

En esta categoría vamos a centrarnos en la visión que tiene el patrimonio en las distintas propuestas didácticas. Para ello, hacemos alusión al tipo de patrimonio que se trabaja, al papel que se le otorga al patrimonio, a los contenidos desarrollados, así como a la tipología del cuento seleccionado.

Con respecto al tipo de patrimonio, dos propuestas didácticas combinan el trabajo del patrimonio histórico, natural y artístico; otras dos propuestas contemplan el patrimonio de manera holística; tres propuestas abarcan el patrimonio etnológico; una propuesta el patrimonio histórico y etnológico; otra propuesta, el patrimonio artístico; y por último, solo una propuesta trabaja el patrimonio natural (Figura 7).

Haciendo referencia al papel del patrimonio en las distintas propuestas (Figura 8), cabe destacar que, en cinco de las diez propuestas, el patrimonio se presenta de manera transversal; en tres de ellas, se utiliza el patrimonio como recurso didáctico; y en dos de ellas, se contempla una integración plena del patrimonio. 


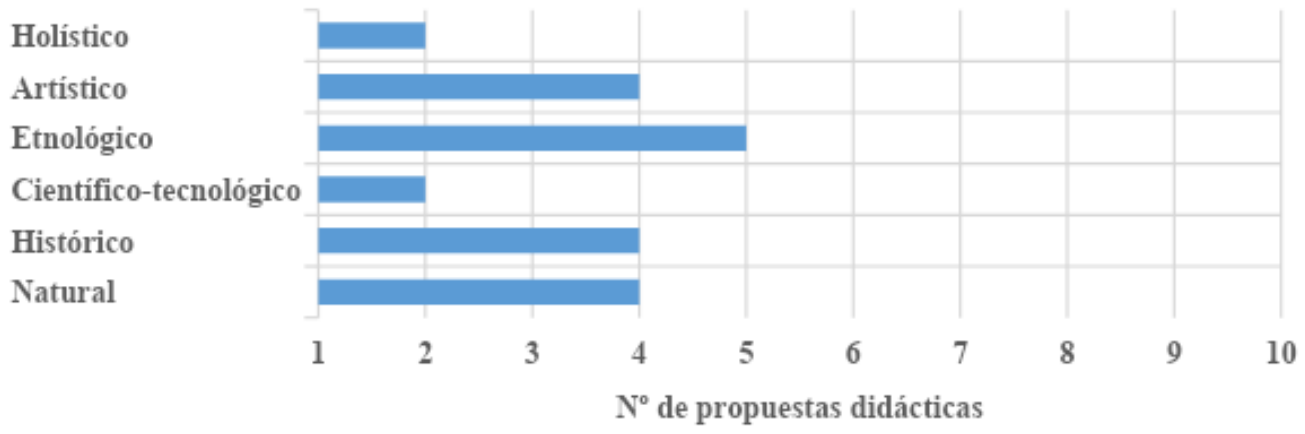

Figura 7. Tipos de patrimonio trabajados en las propuestas didácticas.

Fuente: elaboración propia.

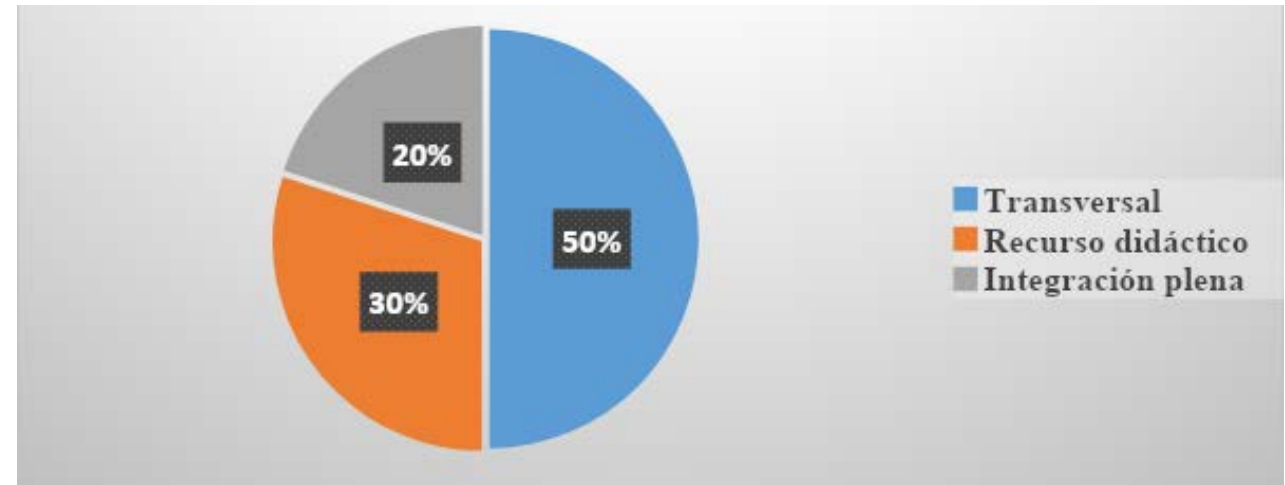

Figura 8. Papel de patrimonio en las propuestas didáctica.

Fuente: elaboración propia.

En cuanto a los contenidos desarrollados en las propuestas didácticas, tal y como se ha comentado en la categoría de la finalidad didáctica, solo dos propuestas contemplan como contenido principal el patrimonio. En estas dos propuestas se trabaja monumentos, lugares y personajes históricos del entorno (Figura 9).

\section{CONTENIDOS}

- Las Haciendas, edificios de valor cultural y tradicional del municipio de Dos Hermanas.

- Historia de Antonia Díaz, J. Lamarque de Novoa y

el conde de Ybarra

- Partes del Parque de la Alquería del Pilar.

- Disfrute de la flora del entorno del parque.

- Poemas andaluces de los personajes trabajados.

- Respeto y valoración del paisaje nazareno.

Figura 9. Ejemplos de contenidos patrimoniales.

Fuente: Propuesta didáctica 1, página 1. 
En el resto de las propuestas, los contenidos patrimoniales también están presentes, como por ejemplo, las costumbres, las tradiciones, las fiestas, o los juegos populares de un lugar en concreto. No obstante, su papel es el de complementar al contenido protagonista de la propuesta didáctica (Figura 10).

\begin{tabular}{|l|l|}
\hline PAÍS & \multicolumn{1}{|c|}{ JUEGOS TRADICIONALES } \\
\hline Marruecos & $\begin{array}{l}\text {-Águir (el asno) } \\
\text {-Hada }\end{array}$ \\
\hline China & $\begin{array}{l}\text {-Cabeza de dragón } \\
\text {-El ratón y el gato }\end{array}$ \\
\hline España & $\begin{array}{l}\text {-El nudo } \\
\text {-La rayuela }\end{array}$ \\
\hline
\end{tabular}

Figura 10. Otros contenidos patrimoniales.

Fuente: Propuesta didáctica 8, página 7.

Por último, en referencia a la tipología de los cuentos utilizados en cada una de las propuestas, se presentan como los más utilizados los cuentos de animales y maravillosos. No obstante, estos cuentos no contemplan contenido patrimonial. Son los cuentos denominados "De costumbres", los cuales han sido elaborados por las propias docentes, los que recogen elementos patrimoniales (Figura 11).

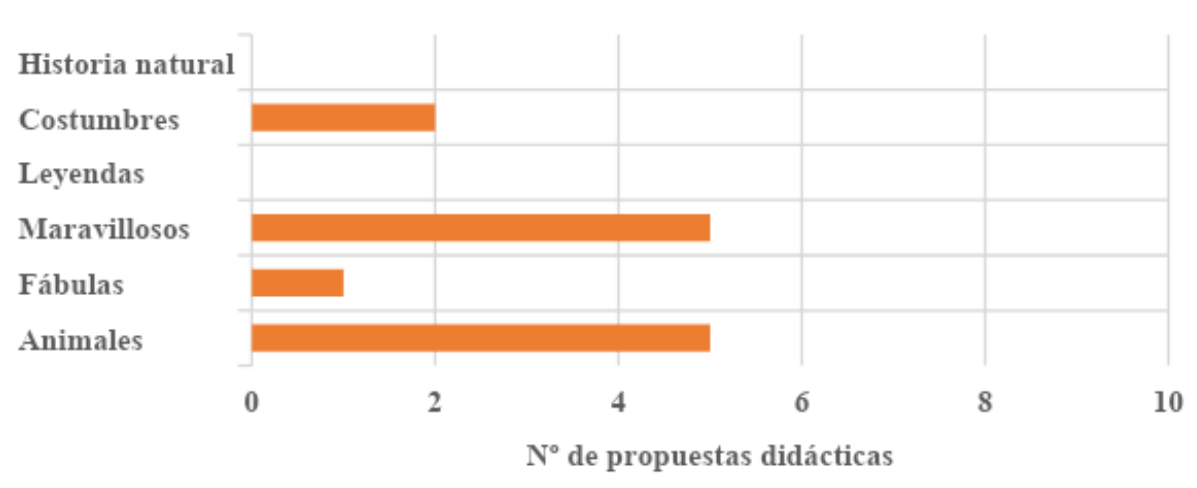

Figura 11. Tipos de cuentos.

Fuente: elaboración propia.

\section{Discusión de resultados}

La cierta novedad de esta investigación aumenta la complejidad de cotejar los resultados obtenidos con otras investigaciones con las que se pudieran comparar, pues a pesar de que la educación patrimonial se está incluyendo cada vez más en esta etapa educativa, no se encuentra un elevado número de estudios que vayan en esta línea. Por consiguiente, se ha llevado a cabo la discusión de los resultados con la fundamentación teórica del informe.

Por un lado, las propuestas didácticas analizadas presentan diferentes métodos para la enseñanza de contenido patrimonial que, según Casanova, Arias y Egea (2018), es el principal 
problema que existe en Educación Infantil, pues al no estar explícitamente incorporado el término de patrimonio en el currículo, la inclusión de estos contenidos queda a merced del docente.

En este mismo sentido, es conveniente hacer referencia a las actividades centradas en la enseñanza de contenido patrimonial. Estas presentan un carácter significativo, globalizador, de descubrimiento, lúdico y motivador para el alumnado, siendo algunas de ellas: visitas a distintos lugares patrimoniales, investigación sobre personajes históricos o sobre la vestimenta de una época pasada, fiestas culturales, creación de obras de artes, entre otras. De este modo, el papel del docente se presenta como un guía del proceso de enseñanza-aprendizaje, y el papel del alumnado como el protagonista de dicho proceso.

Tal y como se recoge en la fundamentación teórica, estos aspectos son apoyados por Miralles y Rivero (2012) como propuestas metodológicas a tener en cuenta en la planificación de la enseñanza y aprendizaje de contenidos sociales en Educación Infantil.

En cuanto al papel que se le otorga al patrimonio en las distintas propuestas, solo en dos de ellas, el docente tiene como objetivo principal la enseñanza de contenido patrimonial; en el resto de las propuestas, el patrimonio está presente pero no como un contenido, sino como un recurso, el cual sirve de ayuda para el desarrollo de otros tipos de contenidos. De este modo, queda reflejada la idea presente sobre la transversalidad del contenido patrimonial en el currículo de Educación Infantil.

Por otro lado, el tipo de patrimonio más trabajado es el etnológico, mediante contenidos relacionados con las costumbres o las tradiciones referentes al entorno cercano del alumnado o de otros países. Según el currículo de esta etapa, desde la escuela se contempla la aproximación del alumnado a los usos y costumbres sociales que les permita conocer diversos modos y manifestaciones culturales presentes en la sociedad.

En el caso de los cuentos infantiles empleados en las propuestas, cabe destacar que los más utilizados son los protagonizados por animales personificados, los cuales no recogen como finalidad la enseñanza de contenido patrimonial; sin embargo, son ellos mismos los considerados como un elemento patrimonial, pues han sido transmitidos oralmente de generación en generación. Dicho análisis coincide con la idea de Morón (2010) sobre que los cuentos infantiles forman parte del repertorio cultural que las generaciones adultas han transmitido oralmente.

Tras haber puesto en antecedente los resultados obtenidos, el siguiente paso es dar respuesta a la problemática de la investigación “QQué criterios se deben de tener en cuenta para desarrollar propuestas de educación patrimonial deseables utilizando los cuentos como recurso didáctico en el aula de 3 a 5 años?". En la Tabla 7, se presentan los parámetros considerados como idóneos para el desarrollo de una propuesta patrimonial deseable: 
Tabla 7

Parámetros idóneos para una propuesta de educación patrimonial.

\section{CATEGORÍAS PARÁMETROS IDÓNEOS}

\begin{tabular}{ll}
\hline Finalidad & Combinar el objetivo didáctico de la propuesta con el resto de objetivos curriculares del \\
didáctica & currículo de Educación Infantil, con el propósito de diseñar actividades en las que se \\
& trabaje de manera globalizadora todos los contenidos requeridos para esta etapa, \\
& incluyendo el contenido patrimonial como una temática transversal a trabajar en la \\
& misma. \\
\hline Metodología & Debido a la diversidad de métodos utilizados por los docentes, lo ideal sería, sin duda \\
& alguna, las metodologías activas, las cuales convierten las aulas en espacios para la \\
& investigación y recreación de los hechos históricos y, por tanto, introducir el conocimiento \\
& histórico en edades tempranas (Miralles y Rivero, 2012). Un ejemplo de metodología \\
& activa sería el Aprendizaje Basado en Proyectos (ABP). \\
& Como estrategias metodológicas principales se contempla el aprendizaje significativo y el \\
& enfoque globalizador. Sin embargo, también son muy importantes, el aprendizaje por \\
& descubrimiento o la participación familiar. \\
& En el caso de las actividades, estas han de propiciar la autonomía, la investigación, el \\
& descubrimiento, la imaginación y la creatividad del alumnado. En este sentido, se \\
& recomienda la realización de asambleas, donde se conozca las ideas previas de los \\
& alumnos y las alumnas, la búsqueda de información autónoma mediante aparatos \\
& electrónicos, y las excursiones o visitas a lugares patrimoniales, con el propósito de que \\
& los niños y ninas tengan un contacto directo con los mismos. \\
& Con respecto al papel del docente, este debe de guiar el proceso de enseñanza-aprendizaje, \\
& otorgándole el papel de protagonista al alumnado, ayudándole y proporcionándole \\
& herramientas didácticas para que construyan su propio aprendizaje. No ha de tener un \\
papel autoritario, pues debido a este, no se tiene en cuenta la opinión de los discentes.
\end{tabular}

Fuente: elaboración propia.

\section{Conclusiones y limitaciones}

Mediante este análisis documental se ha tratado de caracterizar los parámetros idóneos para desarrollar propuestas de educación patrimonial deseables utilizando los cuentos como recurso didáctico en el aula de infantil. Para ello, se ha tenido que dar respuesta a los objetivos específicos de esta investigación:

En el caso de la finalidad didáctica, las propuestas analizadas presentan propósitos diferentes (matemáticos, de lecto-escritura, patrimonio, arte...); no obstante, estos propósitos se combinan con otros objetivos curriculares de la etapa infantil, como son la autonomía, el control corporal, las emociones, la socialización, etc.

En cuanto a los métodos y estrategias, también se observa una diversidad metodológica, pues cada docente decide poner en práctica su propuesta en función a sus ideales o formación profesional. Sin embargo, la mayoría de las actividades analizadas tienen un carácter significativo, globalizador, lúdico, motivador y de descubrimiento.

En este sentido, el papel del docente se muestra como un guía del proceso de enseñanzaaprendizaje y el papel del alumnado como los protagonistas del mismo. A pesar de ello, hay 
aspectos de algunas propuestas que te sugieren lo contrario, como por ejemplo, la realización de actividades más tradicionales y aburridas mediante fichas.

Centrándonos en el proceso evaluativo, las propuestas didácticas plantean los criterios de evaluación en función a los objetivos propuestos, considerando la observación y las producciones del alumnado como instrumentos de evaluación.

Haciendo referencia a los aspectos patrimoniales, en todas las propuestas analizadas se trabajan contenidos patrimoniales pero enfocados de diferente manera: como contenido o como recurso. Además, el tipo de patrimonio más trabajado es el etnológico, no obstante, se comprueba la posibilidad de combinar distintos tipos de patrimonio en una sola propuesta didáctica.

En el caso de los cuentos, los más utilizados son los que tienen como protagonistas animales personificados y presentan elementos fantásticos o maravillosos, pero estos no tienen como objetivo la enseñanza de patrimonio. En este sentido, por un lado, son los cuentos tradicionales los considerados como un elemento patrimonial; y por otro lado, los cuentos denominados "De costumbres", los cuales han sido elaborados por las propias docentes, son los que recogen elementos patrimoniales.

Evidentemente, no existe una fórmula infalible para generar un aprendizaje significativo en todo tipo de alumnado, pues esto depende de muchos factores, como pueden ser la edad, las características individuales o el contexto al que pertenece. Sin embargo, tras esta investigación fundamentada con diversos autores, se ha demostrado la efectividad de una serie de estrategias metodológicas, las cuales se han reflejado en la Tabla 2.

Para concluir, quiero hacer hincapié en que este análisis me ha servido para conocer las características de las prácticas que los docentes de infantil llevan a cabo en sus aulas, descubriendo así las ventajas y desventajas de las mismas. Por consiguiente, la Tabla 7 de parámetros se ha elaborado teniendo en cuenta la información de cada propuesta didáctica que, contrastada con la fundamentación teórica, me ha ayudado a averiguar sus puntos fuertes y débiles, es decir, cuales son los parámetros ideales y cuáles no de una intervención en el aula.

Con respecto a las limitaciones, a la hora de realizar cualquier trabajo de investigación, siempre suele haber ciertos obstáculos que dificultan el estudio a realizar. Dichos obstáculos y limitaciones han estado presente tanto en la planificación previa como en el desarrollo del mismo.

Como limitación principal, de la cual ha derivado los demás obstáculos, se destaca el confinamiento provocado por el COVID-19. En primer lugar, esta pandemia me hizo modificar la organización del trabajo, pues mi pensamiento era la realización de una intervención en el aula sobre patrimonio, pero con el cierre de los centros escolares, me tuve que decantar por llevar a cabo un análisis documental.

En segundo lugar, en relación al marco teórico, prácticamente toda la documentación la he tenido que recabar de internet, mediante documentos digitales tanto de la universidad de Huelva, como de otras bases de datos. No obstante, no he contado con toda la documentación teórica que me hubiera gustado, pues muchos de los libros o artículos pertinentes no se encontraban en formato digital.

Otra de las limitaciones se ha dado durante la búsqueda de las propuestas didácticas. Por un lado, vía internet eran escasas las prácticas en el aula relacionadas con dicha temática, y 
además, las que pude encontrar, contaban con un formato artículo, por lo que no contemplaban toda la información relacionada con la práctica en el aula.

Por otro lado, recurrí a varias docentes para que me ayudaran en la recolección de estas propuestas. En este caso, la mayoría de ellas no contaban con sus programaciones de aula en formato digital, las tenían en formato papel y, además, en el centro escolar.

También, al ser una temática novedosa, solo una de ellas había trabajo patrimonio mediante un cuento, las demás simplemente utilizaban el cuento como un recurso motivador en el aula para empezar una unidad didáctica e, incluso, nunca habían planificado una intervención en el aula para trabajar el patrimonio. Por estos motivos, la muestra de la investigación solo ha sido de 10 propuestas didácticas.

Y como último obstáculo, en relación al formato artículo de las propuestas didácticas, algunas de ellas no me han permitido recabar toda la información que necesitaba para el análisis. Por ejemplo, no contaban con las explicaciones de las actividades o eran demasiado escuetas, lo que ha dificultado la obtención de información sobre el papel del docente y del alumnado. También, la mayoría de ellas, no disponían del apartado de evaluación.

No obstante, a pesar de estos obstáculos y limitaciones, considero esta investigación como un estudio novedoso sobre educación patrimonial en la etapa de Educación Infantil, el cual cuenta con resultados satisfactorios que pueden guiar a aquellos docentes interesados en la temática.

\section{Referencias bibliográficas}

Amar, V. (2018). Déjame que mire un cuento: narración, familia y educación infantil. Una investigación narrativa. Revista de currículum y formación del profesorado, 2(22), 389-405. Recuperado de: https://recyt.fecyt.es/index.php/profesorado/article/view/66379

Arévalo, J.M. (2004). La tradición, el patrimonio, y la identidad. Revista de estudios extremeños, vol. $60,3,925-956$.

Ávila, R.M. (2005). Reflexiones sobre la enseñanza y el aprendizaje del patrimonio integrado. Una experiencia en la formación de maestros. Investigación en la Escuela, 53, 43-53.

Bisquerra, R. (Coord.) (2014). Metodología de la investigación educativa. (4ํㅡㄹ Edición). Madrid: La Muralla.

Casanova, E., Arias, L. y Egea, A. (2018). La metodología por proyectos como oportunidad para la introducción de la historia y el patrimonio en las aulas de Educación Infantil. Contextos Educativos, 22, 79-95. http://dx.doi.org/10.18172/con.3185

Cuenca, J. Ma y Martín, M. (2014). Manual para el desarrollo de proyectos educativos de museos. Gijón: Trea.

Cuenca, J. Mㅜ. (2002). El patrimonio en la Didáctica de las Ciencias Sociales: análisis de concepciones, dificultades y obstáculos para su integración en la enseñanza obligatoria. Tesis Doctoral. Universidad de Huelva. Recuperado de: http://rabida.uhu.es/dspace/handle/10272/2648

Cuenca, J. Mà. (2016). Escuela, Patrimonio y Sociedad. La socialización del Patrimonio. Revista Universidad, Escuela y Sociedad (UNES), 1, 22-41. 
Díaz, M. y Arriaga, C. (2013). Canciones tradicionales en el aula de infantil: en busca del patrimonio heredado. Espacio y tiempo: Revista de Ciencias Humana, 27, 107- 122. Recuperado de: https://dialnet.unirioja.es/servlet/articulo?codigo $=4352041$

Estepa, J., Domínguez, C. y Cuenca, J. Mํa (1998). La enseñanza de valores a través del Patrimonio. En AA.VV. Los valores y la Didáctica de las Ciencias Sociales (pp. 327-336). Lleida: Universidad de Lleida.

Fernández, C.G. (2010). El cuento como recurso educativo. Revista digital Innovación y experiencias educativas, 26, 1-9. Recuperado de: https://archivos.csif.es/archivos/andalucia/ensenanza/revistas/csicsif/revista/pdf/Num ero_26/CRISTINA_GEMA_FERNANDEZ_SERON_01.pdf

Fontal, 0. (2003). La educación patrimonial. Teoría y práctica en el aula, el museo e internet. Gijón: Trea.

Fontal, 0. (2013). La educación patrimonial: del patrimonio a las personas. Gijón. Trea.

Franco, C. y Justo, Eduardo (2009). Efectos de un programa de intervención basado en la imaginación, la relajación y el cuento infantil, sobre los niveles de creatividad verbal, gráfica y motora en un grupo de niños de último curso de educación infantil. Revista Iberoamericana de Educación, 49(3), 1-11.

González, I. (2006). El valor de los cuentos infantiles como recurso para trabajar la transversalidad en las aulas. Revista de Educación "Campo Abierto", 25(1), 11-29. Recuperado de: http://hdl.handle.net/11162/28147.

Guillén, R. y Hernández, A. Ma․ (2018). La colaboración de la escuela y las instituciones culturales para la Educación Patrimonial: estudio de caso. Revista CLÍO: History and History

Teaching, $44 . \quad$ Recuperado de https://dialnet.unirioja.es/servlet/articulo?codigo=6735955.

Jiménez, M. L. y Gordo, A. (2014). El cuento infantil: facilitador de pensamiento desde una experiencia pedagógica. Praxis \& Saber, 5(10), 151-170. Recuperado de: https: / /dialnet.unirioja.es/servlet/articulo?codigo $=5598160$

León, S. (2009). El cuento en Educación Infantil "un mundo de actividades". Revista digital para profesionales de la enseñanza, $1 . \quad$ Recuperado de: https:/ /www.feandalucia.ccoo.es/indicei.aspx?p=62\&d=145\&s=1

Martín, M.J. (2012). La educación y la comunicación patrimonial: una mirada desde el museo de Huelva. Tesis Doctoral. Universidad de Huelva. Recuperado de: http://rabida.uhu.es/dspace/handle/10272/6048.

Miralles, P. y Rivero, P. (2012). Propuestas de innovación para la enseñanza de la historia en Educación Infantil. Revista Electrónica Interuniversitaria de Formación del Profesorado (REIFOP), 15 (1), 81-90. Recuperado de: https://redined.mecd.gob.es/xmlui/handle/11162/94548

Morón, Ma . C. (2010). Los beneficios de la literatura infantil. Revista digital para profesionales de la enseñanza, 8. Recuperado de: https://www.feandalucia.ccoo.es/docu/p5sd7247.pdf Quintero, M. P. (2005). Érase una vez...cuento. Revista Digital de Investigación y Educación. 
Ramón, M. M. (2004). Literatura popular infantil. Una aproximación al cuento como género literario. Revista de la Facultad de Educación de Albacete, 19, 149-159. Recuperado de: https://dialnet.unirioja.es/servlet/articulo?codigo=1264551

Ros, E. (2013). El cuento infantil como herramienta socializadora de género. Cuestiones pedagógicas: Revista de ciencias de la educación, 22, 329-350. Recuperado de: https://dialnet.unirioja.es/servlet/articulo?codigo $=4514882$

Salmerón, P. (2004). Transmisión de valores a través de los cuentos clásicos infantiles. Tesis Doctoral. Universidad de Granada. Recuperado de: https://dialnet.unirioja.es/servlet/autor?codigo=89088 